\title{
Pooling of sera for human immunodeficiency virus (HIV) testing: an economical method for use in developing countries
}

\author{
J C EMMANUEL,* M T BASSETT, H J SMITH,* J A JACOBS* From the *Blood Transfusion \\ Service, Harare, Zimbabwe, and the Department of Community Medicine, University of Zimbabwe
}

SUMMARY To determine whether donated blood samples in African countries could be pooled, then tested for the presence of human immunodeficiency virus (HIV) antibodies with a single test without loss of accuracy, a single test on five pooled samples was used, followed by individual testing of positive pools. This resulted in no loss of either sensitivity or specificity. Pooling 10 samples resulted in a loss of sensitivity for low antibody titre specimens. Pooling reduced the costs of screening by $70 \%$ and time needed for analysis. It is concluded that pooling of five samples for HIV screening may result in a substantial reduction in costs; in countries where the prevalence of HIV is higher than the $2-3 \%$ found in Zimbabwean donors, however, savings may not be as great.

The Blood Transfusion services in Zimbabwe began routine screening for antibody to human immunodeficiency virus (HIV) on all blood donations in July 1985. This practice was adopted because of the well documented risk of HIV infection associated with blood transfusion and has resulted in an apparent low incidence of HIV associated with blood transfusion in this country (unpublished data). The cost of routine screening, however, is high: each screening costs Z\$4.20 (US\$2.59) using the Abbott Laboratories enzyme immunoassay test kit. Each HIV antibody positive specimen is rescreened to confirm seropositivity at an additional cost of $\mathbf{Z} \$ 4 \cdot 20$. The current rate of -HIV seropositivity among donors is $2 \cdot 1 \%$. Thus the average monthly cost to the Harare Blood Transfusion Service, where an average of $\mathbf{4 0 0 0}$ donations are collected, is $\mathrm{Z} \$ 17 \mathbf{1 5 3 . 0 0}$.

Shortly before a screening programme was adopted, we sought to determine whether donated samples could be pooled, then tested with a single test without loss of accuracy. We reasoned that negative pools could then be "cleared" and positive pools tested individually to determine which donation(s) in the pool were HIV antibody positive. This approach would also substantially reduce time devoted to screening because with current rates of seropositivity most pools would be negative. We report here our findings using pools of five and 10 specimens compared with those from individual specimen screening.

Accepted for publication 26 January 1988

\section{Patients and methods}

The screening assay used by the Blood Transfusion Service is the Abbott Laboratories enzyme immunoassay test kit (Human Lymphotrophic Virus Type III EIA). The reliability of this test as a screening tool was established using the Western blot and confirmatory bead EIA as confirmatory tests in laboratories in the United States and West Germany. Three hundred and eighty repeatably reactive specimens and 230 negative specimens were sent for confirmation. West Germany rescreened each specimen using the Abbott enzyme immunoassay before the confirmatory tests were carried out.

Positive and negative specimens were collected from the Harare and Bulawayo Blood Transfusion Services between June 1986 and November 1986.

The enzyme immunoassay results were graded according to absorbance values between + and ++++ . This grading is determined as follows: cut off $=0.500(+) ; 0.501-1.000(++) ; 1.001-1.500$ $(+++) ; 1.501-2.000(++++)$.

\section{Results}

Initially 80 specimens were screened individually and in pools of five and 10. Every pool of five sera identified all of the positive results found by individual screening. Pools of 10 sera, however, did not result in identification of all positive results. The two specimens missed in the 10 pooled sera both had low absorbance values $(+)$. The absorbance values of pools of five and 
Table 1 HIV reactivity from 16 pools of five sera and eight pools of 10 sera, and individual positive results found in each pool

\begin{tabular}{lll}
\hline Pool number & Five sera & $\begin{array}{l}\text { Individual positive sera } \\
\text { in pool }\end{array}$ \\
\hline 1 & ++ & +++ \\
2 & ++ & ++ \\
3 & ++ & +++ \\
4 & +++ & +++ \\
5 & + & ++ \\
6 & ++ & +++ \\
7 & +++ & ++++ \\
8 & +++ & +++ \\
9 & +++ & +++ \\
10 & ++ & +++ \\
11 & +++ & +++ \\
12 & ++ & +++ \\
13 & + & +++ \\
14 & +++ & +++ \\
15 & ++ & +++ \\
16 & +++ & +++ \\
& 10 sera & +++ \\
1 & + & +++ \\
2 & + & ++ \\
3 & + & ++ \\
4 & + & ++ \\
5 & + & ++ \\
6 & + & + \\
7 & + & +
\end{tabular}

pools of 10 sera were generally lower than those found in individual screening (table 1).

This exercise was repeated using $\mathbf{4 4 0}$ sera, or 88 pools of five and 44 pools of 10 sera. Seventeen positive results were identified by the individual testing of all sera. These were also identified in the pools of five sera (17 pools positive or one positive in each pool). Seventeen pools of the 44 pools of 10 sera were
Table 3 Indeterminate results from positive specimens sent for rescreening, confirmatory bead EIA, and Western blot

\begin{tabular}{lllll}
\hline Number & Rescreen & Core & Envelope & Western blot \\
\hline 1 & + & - & - & - \\
6 & + & + & + & + no p24 band \\
2 & + & + & - & + \\
5 & + & - & + & + \\
2 & + & - & + & + no p24 band \\
1 & - & + & + & - \\
1 & - & + & + & + only p24 band \\
1 & + & + & + & - \\
1 & + & + & + & + no p55/65 band \\
1 & + & - & - & + \\
\hline
\end{tabular}

positive. Two specimens with low absorbance values $(+)$ were missed in the pools of 10 .

We calculated costs of screening using the technique of pooling five sera at varying rates of seroprevalence. These calculations do not include the costs of retesting specimens. This is a diagnostic exercise aimed at identifying false positive results, and is not part of the screening process. Savings ranged from 5\% when seroprevalence was set at $15 \%$ to $70 \%$ when seroprevalence was set at $2 \%$. Proportional savings were about the same when repeat screening of positive specimens was performed, although overall costs were higher and rose with increasing seroprevalence (table 2).

Three hundred and eighty repeatably positive specimens were sent to Abbott Reference Laboratories in West Germany for confirmation: 352 $(93 \%)$ specimens were confirmed as positive - that is, rescreen positive, core positive, envelope positive, Western blot positive (all bands). Seven (2\%) specimens were found to be false positive-that is, rescreen negative, core negative, envelope negative, Western blot negative (no bands). Twenty one specimens $(5 \cdot 5 \%)$ had indeterminate results (table 3 ).

Two hundred and thirty negative specimens were

Table 2 Cost savings with pooled sera*

\begin{tabular}{|c|c|c|c|c|}
\hline Prevalence (\%) & Positivity rate per 100 sera $\dagger$ & No of tests done & Total costs & $\%$ reduction in cost with pooling \\
\hline 2 & 2 pools & $\begin{array}{l}20 \text { pools } \\
10 \text { single } \\
\text { Total }\end{array}$ & $\begin{array}{l}\$ 84 \\
\$ 42 \\
\$ 126\end{array}$ & 70 \\
\hline 5 & 5 pools & $\begin{array}{l}20 \text { pools } \\
25 \text { single } \\
\text { Total }\end{array}$ & $\begin{array}{l}\text { \$ } 84 \\
\text { S105 } \\
\$ 189\end{array}$ & 55 \\
\hline 10 & 10 pools & $\begin{array}{l}20 \text { pools } \\
50 \text { single } \\
\text { Total }\end{array}$ & $\begin{array}{l}\$ 84 \\
\$ 210 \\
\$ 294\end{array}$ & 30 \\
\hline 15 & 15 pools & $\begin{array}{l}20 \text { pools } \\
75 \text { single } \\
\text { Total }\end{array}$ & $\begin{array}{l}\$ 84 \\
\$ 315 \\
\$ 399\end{array}$ & 5 \\
\hline
\end{tabular}

*Calculations are based on the assumption that a maximum number of pools will be positive.

†20 pools of five sera per pool. 
Table 4 Indeterminate results from negative specimens sent for rescreening, confirmatory bead EIA, and Western blot

\begin{tabular}{lllll}
\hline Number & Rescreen & Core & Envelope & Western blot \\
\hline 5 & + & - & - & - \\
1 & + & - & - & $+3 \mathrm{p} 32$ bands only \\
1 & - & \pm & - & - \\
5 & - & - & - & + gp41 band only \\
1 & - & - & - & +3 p24 bands only \\
\hline
\end{tabular}

sent, with $215(4 \%)$ specimens confirmed as negativethat is, rescreen negative, core negative, envelope negative, Western blot negative (no bands). Two $(0.9 \%)$ specimens were found to be false negativethat is, rescreen positive and either core, envelope, or Western blot positive. Thirteen (6\%) specimens had indeterminate results (table 4).

\section{Discussion}

Blood transfusion is potentially an important source of HIV infection in Africa. In the high prevalence countries of Uganda, Rwanda, and Zaire the seroprevalence of HIV antibody is reported to be between 8 and $18 \%$, suggesting that the risk associated with unscreened transfusion would be high. ${ }^{12}$

Reduction of this risk is clearly a public health priority, particularly as intervention to change the other major risk factors for transmission of HIV in Africa-heterosexual contact and maternal-infant transmission-will be much more difficult. Unnecessary transfusions must be strongly discouraged. Further reduction in risk can be achieved relatively easily by screening blood supplies for HIV antibody. Unfortunately, the costs of screening blood supplies are high.

The technique described here of pooling five sera reduces screening costs. In Zimbabwe the cost of screening kits with this technique is one third of what it would be if sera were tested individually. This approach is cost effective when the prevalence of seropositivity is $10 \%$ or less. Pooling makes the process of screening somewhat more complex, as records of which sera are pooled must be kept accurately. Such record keeping should be within the capacity of most blood banks. The ability to declare specimens seronegative in lots of five reduces the amount of time spent by technicians on screening.

Our experience with pooling sera for screening purposes differs from those of Milner et $a .^{3}$ They found that aggregates formed when sera were pooled, which resulted in false positive tests. We have found no evidence of these aggregates in our pooling, and our false positive results are probably due to minor nonHIV proteins such as HLA antigens, ${ }^{4}$ antimitochondrial antibodies in the serum, antibodies to nuclear antigens, human leucocyte antigens or human $\mathrm{T}$ cell antigens. The Western blot test identifies an antibody specific for an antigen of known molecular weight but does not identify the antigen bound to the antibody as specific to any particular virus. A positive Western blot specimen shows bands in either the p24 (viral core protein) or gp41 (viral envelope protein) regions of the blot. ${ }^{5}$ Bands in other regions may also be present, but not all positive Western blot specimens provide a complete pattern. A negative Western blot specimen does not have clearly identifiable bands in the p24 or gp41 regions and usually shows a complete absence of any bands on the entire blot strip. ${ }^{4}$

Indeterminate results are influenced by reagents, technique, and individual interpretation. Concentrations of antibody proteins may vary, resulting in lighter bands that may not be clearly visible - that is, indeterminate due to lack of antibody to $\mathrm{p} 24$ or gp41. False positive and negative rates for Western blot and confirmatory bead EIA are not known. ${ }^{5}$

An antigen positive specimen mixed with an antibody positive specimen could result in the neutralisation of one by the other, thus giving a potentially false negative result. As each specimen was tested individually as well as tested in a pool, and as there were no anomalies, it can be assumed that this did not affect the results. When a number of specimens were tested using the Abbott antigen test kit, no antigen was detected, even in cases of AIDS related complex (ARC) or full blown acquired immune deficiency syndrome (AIDS). We were unable to detect any antigen at all in African sera, whether in patients or donors, and the short comings of this test kit when used against African sera has been the subject of other work. It has been shown that HIV antigen appears early and transiently in primary HIV infection. Antibody production follows after which the antigen may disappear. Its reappearance seems to correlate with clinical, immunological, and neurological deterioration. ${ }^{6}$

The false positive rate of $1.8 \%$ and the false negative rate of $0.9 \%$ indicate that the Abbott HTLVIII (HIV) EIA is of adequate sensitivity and specificity when screening blood donations for HIV antibodies in Zimbabwe. Rapid, simple, low cost tests for antibody to HIV are being developed. ${ }^{7}$ None is available yet commercially. Until a less expensive screening test for HIV antibody becomes available, we recommend that pooling of sera in lots of five be adopted in countries where the prevalence of HIV antibody in the donor population is less than $10 \%$.

Acknowledgement is hereby given to IBM for their financial support, to Abbott Laboratories for carrying out the confirmatory testing, and to the staff of the Blood Transfusion Service, Harare. 


\section{References}

1 Mann JM, Francis H, Quinn IC, et al. HIV seroprevalence among hospital workers in Linshasha, Zaire: Lack of association with occupational exposure. JAMA 1986;256:3099-102.

2 Van de Perre P, Lepage P, Kestelyn P, et al. Acquired immunodeficiency syndrome in Rwanda. Lancet 1985;1:336.

3 Milner LV, Vorster BJ, Conradis JD, Brain P. Anti-HTLVIII testing. A practical solution for Blood Transfusion Services? $S$ Afr Med J 1985;68:921-2.

4 Senn DE. Abbott HTLVIII reference laboratory guide to interpretation of Western blot results. Abbott Park, Illinois: Abbott Laboratories, 1985.

5 Burke DS, Redfield RR. False positive Western blot tests for antibodies to HTLVIII. JAMA 1986;256:347.

6 Goudsmit J, Paul DA, Lange JMA, et al. Expression of Human Immunodeficiency Virus antigen (HIV-Ag) in serum and cerebrospinal fluid during acute and chronic infection. Lancet 1986;1:177-80.

7 Carlson JR, Yee JL, Watson-Williams EJ, et al. Rapid, easy economical screening test for antibodies to HIV. Lancet 1987;1:361-2.

Requests for reprints to: Dr JC Emmanuel, The Blood Transfusion Service, PO Box A101, Avondale, Harare, Zimbabwe. 